\title{
Atomic Resolution STEM-EELS Study of Transition Electronic Localization State Induced by Strain.
}

Yuze Gao, ${ }^{1}$ Manuel A. Roldan ${ }^{2,3}$, Jincang Zhang, ${ }^{1}$ Liang Qiao, ${ }^{4}$ Miaofang Chi, ${ }^{2}$ David Mandrus, ${ }^{2,5}$ Xuechu Shen, ${ }^{6}$ Matthew F. Chisholm ${ }^{2}$, David J. Singh ${ }^{2}$, Guixin Cao ${ }^{4,5}$.

1. Department of Physics, Shanghai University, Shanghai 200444, PR China.

2. Materials Science and Technology Division, Oak Ridge National Laboratory, Oak Ridge, TN 37831-6056, USA.

3. Dept. Física Aplicada III \& Instituto Pluridisciplinar, Universidad Complutense de Madrid, Ciudad Universitaria, 28040 Madrid, Spain.

4. Center for Nanophase Materials Sciences, Oak Ridge National Laboratory, Oak Ridge, TN 37831, USA.

5. Department of Materials Science and Engineering, University of Tennessee, Knoxville, TN 37996

6. National Laboratory for Infrared physics, Shanghai Institute of Technical Physics, Chinese Academy of Sciences, Shanghai 200083, People's Republic of China.

Electronic localization in disordered systems is among the most interesting phenomena in condensed matter physics. Depending on the amount, kind of disorder and dimensionality [1], there are two types of localization, classical Anderson localization (AL) and weak localization (WL). The origin of the AL is multiple scattering interference of the waves due to the randomness in the potential, thus altering the nature of the wave functions [2], while WL typically occurs in disordered electronic systems at very low temperatures. Although the WL is generally regarded as a precursor of the AL transition, the underlying physics of this phenomena is not fully understand yet.

To study the transition between weak and strong localization, we synthesize high-quality $\mathrm{ZrO}_{2}$ nanopillars with controlled dimensionality embedded in epitaxial $\mathrm{La}_{2} / 3 \mathrm{Sr}_{1 / 3} \mathrm{MnO}_{3}(\mathrm{LSMO}) / \mathrm{LaAlO}_{3}$ [110] using state-of-art pulsed laser epitaxy. This peculiar nanostructured system offers a great flexibility to manipulate the superficial structural disorder along the interface by controlling the size of $\mathrm{ZrO}_{2}$ nanopillar, thus strongly affects the electronic transport of the LSMO matrix.

To characterize this superstructure and demonstrate the precise control of its electronic properties, we performed a combined study of Electron Energy Loss Spectroscopy (EELS) with the Z-contrast high angle annular dark field (HAADF) imaging technique in the scanning transmission electron microscope (STEM) Nion UltraSTEM 200, a unique tool that allow us to obtain simultaneously composition, chemistry and structure of materials with atomic resolution and sensitivity. These techniques, together with geometric phase analysis allow us to determine the spatial distribution of the epitaxial strain across the samples and clarify its impact on the various electronic localization mechanisms. 
References:

[1] T. Schwartz et al. Nature 446 (2007), p. 52-55

[2] P. Lee and T.V. Ramakrishnan, Rev Mod Phys 57 (1985), p. 287-337

[3] The authors thank S. Dong at Eastsouth University and Z. Gai in ORNL for useful discussions. Work at ORNL was supported by the Department of Energy, Basic Energy Sciences, Materials Sciences and Engineering Division (MAR, LQ, MC, DM, MFC, DJS, GC) and by the European Research Council Starting Investigator Award "STEMOX 239739" (MAR).

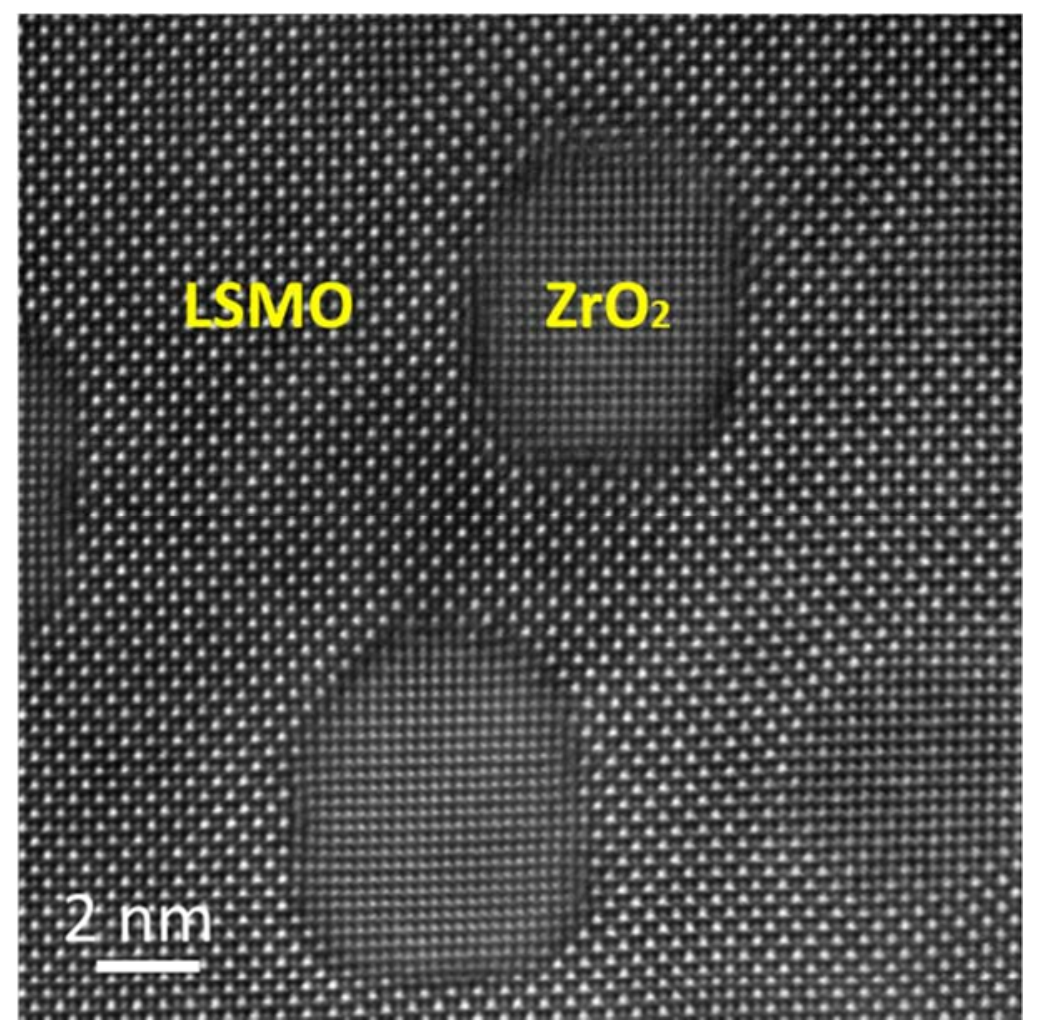

Figure 1. High resolution high angle annular dark field planar view image showing the lattice inside and outside of the $\mathrm{ZrO}_{2}$ circular pillars embedded in LSMO. 